\title{
The pathways from perceived discrimination to self- rated health among the Chinese diaspora during the COVID-19 pandemic: investigation of the roles of depression, anxiety, and social support
}

\section{Youli Chen \\ Wuhan Union Hospital \\ Zicong Wang \\ Wuhan Union Hospital \\ Weizhen Dong \\ University of Waterloo}

Jia Huei Chen Xu

Wenzhou Medical University

Sizhe Ji Wu

Capital Medical University

\section{Xiangyang Zhang}

The First Affiliated Hospital of Wenzhou Medical University

Chun Chen ( $\square$ chenchun408@126.com )

Wenzhou Medical University https://orcid.org/0000-0002-5149-988X

\section{Research Article}

Keywords: Chinese diaspora, COVID-19, self-rated health, perceived discrimination, mental health, social support, cross-sectional, structural equation model

Posted Date: April 26th, 2021

DOI: https://doi.org/10.21203/rs.3.rs-433108/v1

License: (c) (i) This work is licensed under a Creative Commons Attribution 4.0 International License. Read Full License 


\section{Abstract}

Background. Research indicates the adverse impacts of perceived discrimination on health, and discrimination inflamed by the COVID-19 pandemic, a type of social exclusion, could affect the well-being of the Chinese diaspora. The relationship and pathways of perceived discrimination's effect on health among the Chinese diaspora in the context of the pandemic were examined to contribute to the literature on discrimination in this population under the global public health crisis.

Methods. We analysed data from 705 individuals of Chinese descent residing in countries outside of China, who participated in a cross-sectional online survey carried out between April 22 and May 9, 2020. This study utilizes a structural equation model (SEM) to evaluate both direct and indirect effects of perceived discrimination on self-rated health $(\mathrm{SRH})$ and assessed the mediating roles of psychological distress (namely, anxiety and depression) and social support from family and friends.

Results. In this online sample comprised predominantly of young adults and those of relatively high socioeconomic status, this study confirmed the positive and direct effect of perceived discrimination on poor SRH but found the indirect effect was mainly mediated by depression. The association between anxiety and SRH was not significant in this SEM, while a higher level of social support from family leads to better SRH, and the level of social support from friends negatively predicted SRH.

Conclusions. Our findings suggest discrimination negatively affected the well-being of the Chinese diaspora, and depression acted as a major mediator between the discrimination-health relationship. Therefore, interventions for reducing discrimination in order to preserve the well-being of the Chinese diaspora are necessary, and that prompt intervention to address depression may partially relieve the disease burden caused by the surge of discrimination.

\section{Background}

Discrimination is unfair treatment of a perceived group that often results from stigma, or prejudice, which is a negative or hostile attitude based mostly on false or incomplete information (1). A major form of discrimination is based on race; that is, it consists of beliefs, attitudes, and practices that harm individuals or groups because of their physiological features, place of origin, or culture and heritage (2).

Although the Chinese diaspora constitutes a significant part of the world's immigrant population, it is a target for marginalization, stereotyping, and discrimination. In 2007-2008, it was clearly estimated for the first time that the number of Chinese diaspora was approximately 50 million (3), and the number raised to about 69 million in 2017. Apart from 70.4\% of Chinese immigrants living in other Asian countries, the distribution of this population in 2018 was mainly in America (19.6\%), Europe (4.6\%), Oceania (3.2\%), and Africa (2.3\%) (4). Asians, especially those of Chinese descent, face increased incidences of COVID-related racial discrimination, ranging from verbal and physical assaults to vandalism and workplace harassment, regardless of their actual disease status. The situation that Asians 
face today parallels past episodes of discrimination targeted at ethnic minorities related to infectious diseases (5).

The evidence of the cause-and-effect relationship of racism on mental and minority health outcomes is staggering. In a review of 138 empirical studies, Paradies (6) reported that $72 \%$ of the studies found a significant relationship between self-reported racism and mental health and $62 \%$ found physical healthrelated outcomes, including increased risk of cardiovascular, endocrine, and immune response diseases. In the COVID-19 era, various types of racism (e.g., explicit, implicit, institutional, symbolic) are integrative in understanding profound disparities related to COVID-19 (7).

Further, subjective perceptions of self-rated health (SRH) could be used for predicting the abovementioned health trajectories and is a powerful predictor for future mortality and morbidities (8). Prior studies have found a significant link between poorer SRH and perceived racial discrimination in European countries (9) and the US (10). Although the results seem conclusive among various ethnic minorities, including Asians (11), such research specific to the Chinese diaspora is lacking, especially in the context of COVID-19. In this study, we attempt to understand whether subjective discriminatory experiences in the COVID-19 pandemic has negative implications for one's health as measured by SRH. Although the existing literature has focused mainly on the chronic impact of long-term discrimination on health, we assumed that a burst of recent discrimination inflamed by the COVID-19 pandemic could lead to acute health deterioration. This assumption is based on a meta-analytic review done by Pascoe \& Richman (12), which revealed that discrimination that occurred recently (discrimination one has experienced in the past year), compared with long-term discrimination, had a more significant negative effect on mental health, while its relative effect on physical health remained inconclusive.

Although previous literature has left us with in-depth investigation of the directions and pathways of discrimination-health relationships, these results could prove inconclusive in current situations. Thus, this study investigates the association between recent discriminatory encounters and SRH. We utilize SEM to understand the effects of COVID-related discrimination within a biopsychosocial framework, which is widely used in explaining health disparities in minorities $(13,14)$. Specifically, racial discrimination as a stressor elicits physical and psychological responses, and affects mobilization of social resources, which finally leads to health outcomes. In this study, we propose psychological distress (e.g., depression, anxiety) and lack of social support as mediators in the pathway from perceived discrimination to poorer $\mathrm{SRH}$, which have subjected to major changes in the face of the COVID-19 related distancing regulations, the adaptive lifestyles and the pandemic itself.

Many studies point to the negative impact of discrimination on the psychological and social functioning of ethnic minority persons. There is preliminary evidence that psychological distress can mediate the relationship between perceived discrimination and health conditions. One study found that such a relationship could be understood through indirect effects of psychological mediators, including elevated stress and depressive symptoms (15). Notably, the Chinese diaspora is suffering from prominent stigma- 
associated psychological stress during the COVID-19 pandemic (16), and the role of mental health status in the discrimination-health relationship should be investigated promptly.

As demonstrated in previous literature (17), social support is closely associated with general health and $\mathrm{SRH}$. A recent study pointed out that mandatory isolation during the COVID-19 pandemic left older adults more vulnerable to physical and cognitive decline resulting from loneliness $(18,19)$. There is also a strong rationale for expecting that everyday discrimination will lead to decreased social support, probably caused by a limitation of one's social interactions to avoid repeatedly experiencing discrimination (20). During COVID-19, the perceived decrease in social support could be exacerbated by an objective decrease because of the requirement for 'social isolation' and hypervigilance in interpersonal contact for COVID self-protection. It is noteworthy that during the COVID-19 pandemic, social support from friends and family could be widely heterogenous, because home-restraining regulations make people housebound and mostly reduce people's interaction with friends, whereas domestic activities remain intact.

Aside from the above major factors incorporated in this conceptual framework, socio-demographic characteristics have also been recognized as significant determinants of health. A large body of literature on a wide variety of samples has found an association between SRH and socio-demographic characteristics. Female gender, older age, a lower level of education (21), lower income (22), not being married or living alone (23), were important factors associated with general health in European countries. Specific to immigrants, older age and a lower level of attained education were found to be associated with poor SRH in female Ukraine immigrants in Czechoslovakia (24). Hong \& Lee (25) found SRH was income-related and associated with the type of migration. Given the specific context of COVID-19, variations in the policy regarding containment and closure, including stay-at-home orders and policies limiting daily activities and gatherings, could also exert an influence on the accessibility of social support as well as mental health status. Thus, demographic, and psychosocial factors were included in this model to control for their potentially confounding effects on other concepts in the analytic framework.

Using a conceptual framework based on the biopsychosocial theory of health specific to the COVID-19 context, we propose the following research hypothesis (Fig. 1):

\section{Hypothesis 1}

Perceived discrimination during the COVID-19 pandemic has a direct and negative impact on SRH.

\section{Hypothesis 2}

Beyond the direct and negative association with SRH, perceived discrimination influences SRH indirectly, via psychological distress, namely, anxiety and depression. Specifically, anxiety and depression are consequences of discrimination, and contribute to the negative assessment of SRH.

\section{Hypothesis 3}


After controlling for other covariates, the relationship between perceived discrimination and $\mathrm{SRH}$ is mediated by social support from family and friends. Explicitly, we expect the level of social support from friends to decrease when perceived discrimination increases, and the lack of both sources of social support (family and friends) would lead to lower SRH.

Sparse literature has focused on the health consequences and mechanisms of spiking discrimination toward Chinese during the pandemic, although there have been calls for awareness of stigma, and incorporation of coronavirus-related measures or methods into studies. This pandemic is a historical moment that will have a lasting effect on interpersonal and intercultural relations, which merits in-depth research (26).

The current study was carried out in the first-wave, early, escalating stage of the COVID-19 pandemic. As the pandemic evolves, several countries are taking a hit of the second-wave pandemic already, which indicates the inevitability of prolonged co-existence of humans and the coronavirus. Improved understanding of the mechanisms underlying the discrimination-health association would be valuable to inform intervening efforts to reduce stigma and disparities towards ethnic minorities (27), such as those of Chinese descent, and equipping the world to better adapt to public health challenges.

\section{Methods}

\section{Data}

Data in this study were collected by a multi-country, cross-sectional online survey of the Chinese diaspora residing in countries during the Covid-19 pandemic. Respondents were from all continents (except for Antarctica), which included 'epicenter' countries (e.g., the US, the UK, Spain, etc.) and others (e.g., Japan, Australia, etc.). The survey was carried out between April 22 and May 9, 2020.

The study sample was formed by a combination of snowball sampling and random sampling. Based on an online crowdsourcing platform powered by www.wenjuan.com, a part of the survey sample was collected from participants by targeted snowball sampling using a dominant social media platform in China, WeChat, with a link of the online questionnaire attached to each invitation. In addition, the survey collected a random sample using the corporate mailing list of dingwei.netease.com, a survey company, via e-mail with a link to the web-based survey. Among the mailing list, 3,194 of approximately $1,000,000$ users who fitted the requirement for participation in the survey were randomly selected and sent e-mails. A total of 1,045 respondents completed the survey (328 from snowball sampling and 717 from random sampling). Afterward, the invalid questionnaires were screened by C.Y. (missing values $\geq 15 \%$ of question items, answering time $\leq 3$ seconds per question item), which resulted in a valid analytic sample comprised of 705 responses.

\section{Measures}

\section{$\mathrm{SRH}$}


The end outcome variable, $\mathrm{SRH}$, was obtained by a single item asking participants to rate their general health status on a vertical, 0-100-point visual analogue scale (VAS) as part of the Euroqol 5-Dimension Health Questionnaire (EQ-5D)(28). The VAS rated the health status with endpoints of best state set at 100 and worst state set at 0 . EQ-5D is a psychometrically sound tool and currently is being widely used by researchers in a variety of clinical fields and geographical locations (29).

\section{Perceived discrimination}

The latent perceived discrimination construct was comprised of five items adopted from the Everyday Discrimination Scale (EDS) (Short Version) (30) and modified to apply to the COVID-19 pandemic setting (Supplementary Table 1). The original study used this scale in a community sample of black, Hispanic, and white adults and achieved acceptable reliability (Cronbach's $a=0.77$ ). This scale was selected because it is a widely used, validated measure in identifying ethnically different causes and consequences of perceived discrimination in Asians $(31,32)$.

In our modified version, the construct describes five examples of discrimination (e.g., treated with less courtesy/respect than other people poorer service than others at restaurant/store) and asked respondents to indicate how often they encountered these situations in their everyday life since the outbreak of COVID19 based on a 5 -point Likert scale ( $0=$ never, $4=$ always $)$. The five items of perceived discrimination had an internal reliability of 0.89 , and removing any of them would reduce Cronbach's Alpha Coefficient.

\section{Anxiety and depression}

Anxiety and depression were dichotomous variables. Specifically, the Generalized Anxiety Disorder Scale (GAD) -2 and the Patient Health Questionnaire (PHQ) -2 were used to assess the presence of anxiety and depressive symptoms experienced by participants over the past two weeks (33), separately. The GAD-2 included the items 'feeling nervous, anxious, or on edge' and 'not being able to stop or control worrying'. The PHQ-2 included the items 'little interest or pleasure in doing things' and 'feeling down, depressed, or hopeless'. Responses to each item were coded as $0=$ not at all, $1=$ several days, $2=$ more than half the days, and $3=$ nearly every day. Scores of each scale were summed with a potential range of 0 to 6 , where a higher score indicated a higher degree of anxiety or depression. In this study, both the GAD-2 (Cronbach's $a=0.83$ ) and PHQ-2 (Cronbach's $a=0.75$ ) achieved acceptable reliability. We further dichotomized anxiety or depression as 1 when the scale score was $\geq 3$, otherwise as 0 , based on the suggested cut-off point by Kroenke et al. (33), where a scale score $\geq 3$ suggested a likely condition of anxiety or depression. These scales were developed to screen for depression and anxiety disorders and were selected for their brevity and high sensitivity and specificity (34). They were validated and standardized in the general population in Germany (34) and Columbia (35), and in healthcare workers in China (36), etc.

\section{Social support}

Social support is a latent construct measured by 6 modified items adopted from the LSNS- 6 . Both the original version and the modified version consisted of subscales considering social contact with family 
members and friends. The response categories were none, one, two, 3 or $4,5-8$, and 9 or more, which were assigned scores of $0-5$, respectively. Compared with the original scale, both online or in-person social contact were included to fit the current pandemic and the widened age range of participants. Although this scale has been commonly used among community-dwelling elders because Lubben found that marital status and participation in religious activities vary less in the older adult population.

Therefore, this measure focuses more heavily on the quality and frequency of an individual's relationships with family and friends $(37,38)$. This brief instrument fitted our purpose to specifically and separately gauge objective social isolation as well as perceived social support from family and friends, interactions with whom subject to drastic change during the pandemic. The composite reliability of the modified LSNS- 6 was 0.828 , and the reliability of the questions for the family and the friend subscales were 0.75 and 0.81 , respectively.

\section{Socio-demographic characteristics}

A set of socio-demographic characteristics were included in the model as control variables in each structural equation.

Dichotomous variables include gender ( $1=$ male, $0=$ female), location of residence $(1=$ Asian countries, 0 = Non-Asian countries), employment status ( 1 = currently employed [employed full-time/part-time/selfemployed], 0 = currently unemployed [student/retired/unable to work]), marital status ( $1=$ married [common law or legally] or living with a partner, $0=$ other statuses [e.g., single, divorced, or others]), and immigration status ( 1 = non-immigrant, $0=$ immigrant [citizen/LPR [legal permanent resident]/CPR [conditional permanent resident]. Age was an ordinal variable. Educational level was categorized into three ordinal groups: $\leq 12$ years, $\leq 15$ years, and $>15$ years.

An indicator for stringency of government responses to COVID-19 was proposed by Oxford University, which quantifies variation in containment and closure policies, including stay-at-home orders and policies limiting daily activities and gatherings (39). We adopted policy stringency in the proposed model as a continuous variable for its potential impact on health and social support parameters. Respondents were assigned a 0-100 stringency index number corresponding to their location on April 22nd, as calculated by the Oxford COVID-19 Government Response Tracker (OxCGRT).

\section{Data analysis}

First, descriptive statistics were generated for all variables. Normality was tested using skewness and kurtosis of distribution (40). In the analysis, either an absolute skew value larger than 2 or an absolute kurtosis larger than 7 were used as reference values for determining substantial deviation from normality. The original scores of all variables were all normally distributed.

Next, to explore the relationships among the study variables, we first entered all proposed measures into a correlation matrix to identify significant bivariate relationships among them. Two-tailed tests were utilized $(p \leq 0.05)$ to identify significant associations. If a variable was directly correlated with any of the 
variables on the left-hand side or variables were significantly associated with variables on the left-hand side, this information was used to develop an initial, hypothesized model.

Finally, the main analysis was a multiple mediator analysis. The models were analysed using Mplus Version 7 (41). To test our framework in Fig. 1, a structural equation model (SEM) with latent variables was utilized to test the 3 hypotheses simultaneously. Each model was adjusted for gender, age, location of residence, policy stringency, educational level, employment status, marital status, and immigration status. Indirect effects were tested using a bootstrapping procedure using 5,000 resamples from the data set.

This study followed Muthén \& Muthén (41) to report model-fit indices including Chi-square/df, Comparative Fit Index (CFI), the Tucker-Lewis Index (TLI), and the Root Mean Square Error of Approximation (RMSEA). Chi-square/ $\mathrm{df}<3$ indicates an acceptable fit. RMSEA values of 0.08 or lower are indicative of a good fit. CFI values greater than roughly 0.90 may indicate a reasonably good fit of the model, though a value of 0.95 is preferable. TLI values of 0.90 or higher indicate incremental fit, and TLI values exceeding 0.95 indicate good model fit $(42,43)$. We improved model fit during the estimation process by freeing covariances between error terms of the 5 measures of perceived discrimination and 6 measures of social support.

Six structural equations were specified with each variable on the left-hand side of the equation corresponding to one of the six key endogenous variables identified in the conceptual framework depicted in Fig. 1.This study followed a two-step estimation procedure as suggested by Anderson and Gerbing (44). In the first step, confirmatory analysis and model fit statistics were used to establish an acceptable measurement. In the second step, the modified measurement model and the structural equations were estimated simultaneously. We employed the mean- and variance-adjusted weighted least squares (WLSMV) with listwise deletion of missing values as the method of estimation in both steps. When categorical outcome variables or a mixture of binary, ordinal categorical, and continuous outcome measures are included in SEM, estimation methods designed for categorical variables are recommended for less bias and in terms of model-data fit (45). Listwise deletion for missing values was adopted because missing values relating to sociodemographic variables are not random, and any filling-in method could cause false estimations.

This study intended to assess theoretical and context-based hypothesized associations and pathways between perceived discrimination and $\mathrm{SRH}$, and to explore the associations between the demographic variables and perceived discrimination, psychological distress, social support, and SRH. Thus, the study design consisted of both exploratory and confirmatory data analysis.

\section{Results}

\section{Descriptive statistics}


Descriptive data of all manifest variables in the analysis are summarized in Table 1. A total of 705 individuals completed the survey. Among respondents, $52.3 \%$ were males, $83.4 \%$ were currently living outside of Asia, $78.0 \%$ of them had received some post-secondary education (> 12 years), and $36.5 \%$ were married or living with a partner. $41.9 \%$ of participants were employed currently. As for immigration status, $46.1 \%$ were citizens, legal permanent residents, or conditional permanent residents. The majority of respondents were $18-40$ years of age (83.1\%). 
Table 1

Descriptive statistics of the manifest variables obtained from the survey

\begin{tabular}{|c|c|c|}
\hline & N, No. (\%) & Mean, mean $\pm S D$ \\
\hline $\mathrm{SRH}^{\mathrm{a}}(0-100)$ & $705(100 \%)$ & $85.97 \pm 13.06$ \\
\hline Perceived discrimination & 705 (100\%) & \\
\hline $\mathrm{PD} 1^{\mathrm{b}}(0-4)$ & 705 (100\%) & $2.14 \pm 1.00$ \\
\hline $\mathrm{PD} 2^{\mathrm{b}}(0-4)$ & 705 (100\%) & $1.88 \pm 0.98$ \\
\hline $\mathrm{PD}^{\mathrm{b}}(0-4)$ & 705 (100\%) & $2.02 \pm 1.05$ \\
\hline $\mathrm{PD} 4{ }^{\mathrm{b}}(0-4)$ & 705 (100\%) & $2.02 \pm 1.03$ \\
\hline $\mathrm{PD} 5^{\mathrm{b}}(0-4)$ & 705 (100\%) & $1.73 \pm 0.95$ \\
\hline Anxiety $(0=\mathrm{NO}, 1=\mathrm{YES})$ & $705(100 \%)$ & $0.3 \pm 0.46$ \\
\hline 0 (REF) & $495(70.2 \%)$ & \\
\hline 1 & $210(29.8 \%)$ & \\
\hline Depression ( $0=$ NO, $1=$ YES $)$ & $705(100 \%)$ & $0.26 \pm 0.44$ \\
\hline 0 (REF) & $525(74.5 \%)$ & \\
\hline 1 & $180(25.5 \%)$ & \\
\hline Social support & 705 (100\%) & \\
\hline Social support from family & 705 (100\%) & \\
\hline $\mathrm{SS}^{\mathrm{C}}(0-5)$ & 705 (100\%) & $3.69 \pm 1.35$ \\
\hline $\mathrm{SS} 2^{\mathrm{C}}(0-5)$ & 705 (100\%) & $3.13 \pm 1.32$ \\
\hline$S S 3^{c}(0-5)$ & 705 (100\%) & $3.51 \pm 1.31$ \\
\hline Social support from friends & $705(100 \%)$ & \\
\hline $\mathrm{SS} 4^{\mathrm{C}}(0-5)$ & $705(100 \%)$ & $4.00 \pm 1.36$ \\
\hline$S S 5^{c}(0-5)$ & 705 (100\%) & $3.48 \pm 1.30$ \\
\hline$S S 6^{c}(0-5)$ & 705 (100\%) & $3.58 \pm 1.38$ \\
\hline Gender & $701(99.4 \%)$ & $0.53 \pm 0.50$ \\
\hline
\end{tabular}

(a) SRH: self-rated health; (b) PD: perceived discrimination; (c) SS: social support; (d) LPR: legal permanent resident; (e) CPR: conditional permanent resident 


\begin{tabular}{|c|c|c|}
\hline & N, No. (\%) & Mean, mean \pm SD \\
\hline Male & $369(52.3 \%)$ & \\
\hline Female (REF) & $332(47.1 \%)$ & \\
\hline Age & $700(99.3 \%)$ & $2.88 \pm 1.26$ \\
\hline$<18$ & $36(5.1 \%)$ & \\
\hline $18-25$ & $324(46.0 \%)$ & \\
\hline $26-30$ & $145(20.6 \%)$ & \\
\hline $31-40$ & $116(16.5 \%)$ & \\
\hline $41-50$ & $52(7.4 \%)$ & \\
\hline $51-60$ & $16(2.3 \%)$ & \\
\hline$>60$ & $11(1.6 \%)$ & \\
\hline Location of residence & & $0.17 \pm 0.37$ \\
\hline Asian countries & $117(16.6 \%)$ & \\
\hline Non-Asian countries (REF) & $588(83.4 \%)$ & \\
\hline Policy stringency, mean (SD) & $704(99.9 \%)$ & $76.13 \pm 13.27$ \\
\hline Educational level & $689(97.7 \%)$ & $2.27 \pm 0.78$ \\
\hline$\leq 12$ years & $139(19.7 \%)$ & \\
\hline$\leq 15$ years & $227(32.2 \%)$ & \\
\hline \$15 years & $323(45.8 \%)$ & \\
\hline Employment status & $683(95.5 \%)$ & $0.51 \pm 0.5$ \\
\hline Currently employed (employed full-time/part-time/self-employed) & $346(49.1 \%)$ & \\
\hline Currently unemployed (student/retired/unable to work) (REF) & $337(47.8 \%)$ & \\
\hline Marital status & $673(95.5 \%)$ & $0.38 \pm 0.49$ \\
\hline Married/ Living with a partner/ Common law & $257(36.5 \%)$ & \\
\hline Unmarried (Single/Other) (REF) & $416(59.0 \%)$ & \\
\hline Immigration status & $668(94.8 \%)$ & $0.51 \pm 0.50$ \\
\hline Immigrant (Citizen/LPR $\left.{ }^{\mathrm{d}} / \mathrm{CPR}^{\mathrm{e}}\right)$ (REF) & $325(46.1 \%)$ & \\
\hline
\end{tabular}

(a) SRH: self-rated health; (b) PD: perceived discrimination; (c) SS: social support; (d) LPR: legal permanent resident; (e) CPR: conditional permanent resident 


\section{N, No. (\%) Mean, mean \pm SD}

Non-immigrant

$343(48.7 \%)$

(a) SRH: self-rated health; (b) PD: perceived discrimination; (c) SS: social support; (d) LPR: legal permanent resident; (e) CPR: conditional permanent resident

Participants' mean score of SRH is $85.97 \pm 13.06$. Anxiety (29.8\%) and depressive symptoms $(25.5 \%)$ were relatively prevalent among participants.

\section{Correlation matrix}

The significant associations identified in Supplementary Table 3 indicate that SRH was negatively associated with all 5 manifest variables in the perceived discrimination construct, anxiety, and depression, and positively associated with frequent contact with family members and the number of trusted relatives. Also, the 5 manifest variables in the perceived discrimination construct were positively associated with the likelihood of anxiety and depression. Manifest variables in the perceived discrimination construct and manifest variables in the social support construct of statistical significance all indicate converse relationships.

Outside the variables in our initial hypotheses, we also found SRH negatively associated with educational level. Older people, those currently employed, and married people were more likely to have anxiety.

\section{SEM Results}

\section{Initial model}

In the analysis of the initial path model (Fig. 1), goodness-of-fit indices for the original model indicated a low degree of fit (Chi-square $/ \mathrm{df}$ value $=2.526 ; \mathrm{CFI}=0.893 ; \mathrm{TLI}=0.840 ; \mathrm{RMSEA}=0.049$ ). All standardized factor loadings of manifest variables in latent constructs were nontrivial and significant, which were preserved in the final model (Supplementary Table 4). Several paths in the model were not statistically significant: from perceived discrimination to social support from family $(p=0.057)$, and from anxiety to $\mathrm{SRH}(p=0.808)$. However, given that the pathway coefficient from perceived discrimination to social support from family is of marginal significance, and the conceptual importance of anxiety in the model, all paths were retained in the final model.

Revised model

A robust measurement model was established after freeing covariances between error terms of variables in latent constructs, with several model fit statistics indicating a good model fit of the measurement model. Specifically, the Chi-square/df value $(241.322 / 133=1.81)$ for the model was lower than 3 , the CFI value (0.944) and the TLI value (0.915) for the model were higher than 0.90 , and the RMSEA value $(0.036)$ was lower than 0.05 , suggesting a good fit of the model. Table 2 illustrates standardized path coefficients corresponding to Fig. 2, the variables on the left-hand side being endogenous variables: perceived 
discrimination, depression, anxiety, social support from family and friends, and SRH. Substantive findings are summarized as follows.

Table 2

Standardized path coefficients from the SEM
Variables on the left-hand side
(1)
(2)
(3)
(4)
(5)
$\mathrm{SRH}^{\mathrm{a}}$

Variables on the right-hand

side $\downarrow$
(1) Perceived discrimination
$0.442^{\star \star \star}$
$0.411^{\star \star \star} \quad-0.098$
$-0.124^{\star \star}$

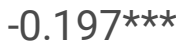
(2) Depression
$-0.247 * \star$
(3) Anxiety
0.022
(4) Social support from family
$0.187^{\star *}$
(5) Social support from
$-0.129 *$

\section{friends}

\section{Sociodemographic characteristics}

\begin{tabular}{|c|c|c|c|c|c|c|}
\hline Male & $0.106^{*}$ & -0.077 & -0.029 & -0.074 & $-0.114^{\star}$ & 0.023 \\
\hline Age & $-0.237 * \star \star$ & -0.091 & 0.100 & $0.173^{*}$ & 0.099 & -0.067 \\
\hline Location of residence & 0.001 & 0.092 & -0.008 & -0.066 & $-0.102^{\star}$ & $0.091 *$ \\
\hline Policy stringency & -0.061 & 0.078 & 0.063 & 0.057 & 0.051 & 0.043 \\
\hline Educational level & -0.012 & -0.015 & -0.077 & 0.042 & $0.162^{\star \star}$ & -0.014 \\
\hline Employed & $0.126^{\star}$ & $<0.0001$ & 0.058 & 0.051 & 0.042 & 0.02 \\
\hline Married & $0.150^{\star}$ & 0.081 & 0.058 & 0.130 & -0.033 & -0.082 \\
\hline Non-immigrant & 0.035 & 0.021 & $0.154^{\star}$ & 0.099 & 0.067 & -0.083 \\
\hline \multicolumn{7}{|c|}{$\star, p<0.05 ; * \star, p<0.01 ; * \star \star, p<0.001$} \\
\hline \multicolumn{7}{|c|}{ (a) SRH: self-rated health } \\
\hline
\end{tabular}

First, we found modulating effects of several sociodemographic factors on endogenous variables. Males, younger people, the employed, and married people reported more encounters of discrimination.

No sociodemographic characteristics were found correlated to depression, while non-immigrants were more likely to exhibit anxiety symptoms. After accounting for sociodemographic characteristics, perceived discrimination significantly led to depression and anxiety, lending support to Hypothesis 2 .

The fourth and fifth columns demonstrate the results using social support as the dependent variable. A higher level of social support from family was anticipated by older age, while females, people living 
outside of Asia, and people who attained a higher level of education possessed more friendship-related social support. Perceived discrimination was found to be, as expected, negatively associated with social support from friends, which partially supported our Hypothesis 3.

Finally, SRH was the dependent variable in the sixth column that allowed us to examine the remaining hypotheses. First, after accounting for other covariates, perceived discrimination was found negatively associated with $\mathrm{SRH}$, which is in accordance with Hypothesis 1. Second, the negative relationship between depression and SRH partially confirmed our Hypothesis 2 but the association between anxiety and SRH was not significant in this SEM. Third, a higher level of social support from family leads to better $\mathrm{SRH}$, and the level of social support from friends negatively predicted $\mathrm{SRH}$, partly contradicting our Hypothesis 3. Also, living in non-Asian countries was found to be positively associated with better SRH.

The direct and indirect effects of perceived discrimination on SRH are summarized in Table 3. Overall, both direct and indirect effects of perceived discrimination on SRH were significant. The magnitude of direct effect (-0.197) was higher than indirect effect (-0.102), indicating perceived discrimination's strong and direct impact on SRH. The indirect effect was solely significantly contributed to depression (-0.109). Social support from either friends or family did not exhibit significant mediation effect on SRH. Although the level of social support from friends was found to be negatively associated with perceived discrimination, its effect on SRH was negative, which could account for the finding that indirect effect through social support from friends was not statistically significant. However, when indirect effects were tested by bootstrapping method, family-related support presented with potential negative mediating effects, while friendship-related support presented with potential positive mediating effects, which warrants future investigation. While perceived discrimination negatively contributed to anxiety, the effects of anxiety on SRH were not significant, which could account for the finding that indirect effects through anxiety were not significant.

Table 3

Summary of standardized direct and indirect effects of discrimination on SRH

\begin{tabular}{|lllll|}
\hline & & Coefficient & p-values & $95 \% \mathrm{Cl}$ \\
\hline Total effect & -0.299 & $<0.001$ & $(-0.392,-0.198)$ \\
\hline Direct effect & -0.197 & $<0.001$ & $(-0.295,-0.095)$ \\
\hline \multirow{2}{*}{ Total indirect effect } & & -0.102 & 0.001 & $(-0.165,-0.046)$ \\
\hline \multirow{2}{*}{ Indirect effect via } & Depression & -0.109 & 0.004 & $(-0.188,-0.041)$ \\
\cline { 2 - 5 } & Anxiety & 0.009 & 0.788 & $(-0.057,0.076)$ \\
\cline { 2 - 5 } & Social support from family & -0.018 & 0.128 & $(-0.052,-0.002)$ \\
\cline { 2 - 5 } & Social support from friends & 0.016 & 0.127 & $(0.002,0.044)$ \\
\cline { 2 - 5 }
\end{tabular}

\section{Discussion}


This study was the first to attempt to attain the SRH of the Chinese diaspora during the COVID-19 pandemic, to examine the associations of perceived discrimination and $\mathrm{SRH}$ in this sample and to assess whether this relationship was mediated by psychological distress and social support. Results yielded by the SEM indicate that perceived discrimination was both directly and indirectly associated with $\mathrm{SRH}$, and that depression was a significant mediator of this relationship. This study adds to a growing literature in this area and extends findings supporting a link between recent discriminatory experiences and health deterioration among the Chinese diaspora, specific to the COVID-19 context. This is an important extension, as Asian and Chinese populations have been faced with a surge of discrimination since the COVID-19 outbreak, and this could pose challenges in areas relating to public health in the future. Moreover, the existence of significant relationships was established despite the particular characteristics of the current sample, which included participants who were relatively well-educated (46) and possibly of relatively high socioeconomic status. Notably, socio-demographic variables rarely affected SRH. These results reflected on the ubiquity of racial discrimination and its impact on health, especially in the COVID19 context.

Another potential contribution of this study lies in its findings of indirect effects. In this biopsychosocial model, the indirect effect of discrimination on health was found to be mediated by depression, which accounted for over $1 / 3$ of the estimated variance of the association. This result echoed the conclusion of some previous studies on ethnic minorities, which assessed psychological distress, and predominantly depression, as a mediator of the discrimination-health relationship $(15,47)$. It is possible that the implicit instead of overt nature of modern-day discrimination is associated with rumination, which is an important process in the development, sustenance, and recurrence of depression (48) and prolongs the physiological responses to sources of stress sources, such as discrimination (49). However, while there was a significant link between perceived discrimination and anxiety, similar to the findings of existing studies (50), anxiety was not a significant mediator between the discrimination-health relationship in this SEM, unlike in a previous study on African-American women (51). A possible explanation could be the low degree of somatization of mental disorders among the Chinese. Assari (52) found ethnic variations in the correlations between mental health issues and $\mathrm{SRH}$, where no significant correlations were found between anxiety and SRH among Chinese immigrants. Although emphasis has been put on investigating and preserving mental health of minority groups subject to discrimination in current context of the pandemic, this study adds yet another piece of evidence for mental health's importance in not only itself, but also its effect on general health as a linkage in the discrimination-health relationship.

Furthermore, social support measured by the number of family members or friends participants frequently interacted with did not display a significant mediating effect on the relationship between perceived discrimination and health in the COVID-19 context. When deconstructed, discrimination did not affect social support from family members, which was obviously logical. Nevertheless, the potential health-promoting effect of social support from family members should be noted and utilized. However, while social support from friends correlated negatively with perceived discrimination, indicating a certain level of social withdrawal because of perceived discrimination among participants, it was associated negatively with $\mathrm{SRH}$, contradicting the hypothesized buffering effect of social support from friends. This 
could be due to hostile or cynical responses provided by friends when participants were seeking social support, which may diminish the health benefits of social support (53). Another explanation lies in the irrationality of contending that social interaction and social support are inherently good (54). Increased conflict or shared health-compromising behaviors (e.g., tobacco and alcohol use) in social networks may result in adverse health outcomes (55).

However, several limitations of this study should be noted and the results be interpreted with caution. First, though the SEM in this study is recursive in nature, the present study was cross-sectional, and definitive causal relationships await longitudinal study designs. Second, the online survey sample was not entirely randomized, yielding results subject to coverage and volunteer bias. For example, young adults (18-25 years of age) were likely over-represented, comprising nearly half of the sample. However, despite these limitations, we managed to produce an extrapolatable sample of the Chinese diaspora with international coverage in a timely fashion that also adequately met the sample size requirements for SEM (56), although the Chinese diaspora is among the populations that are difficult to sample (57). Last, the hypothesized mediators in this study were not exhaustive, although it fits roughly into the framework of and the measures used in this study are limited. For example, past research has revealed that perceived discrimination also contributes negatively to SRH by lowering an individual's social capital (20), and that coping style and ethnic identity also moderate the link between perceived discrimination and health (12). Future research could consider including other potentially influential covariates, moderator, and mediator variables, or adjusting the measurements used in this study.

\section{Conclusions}

Although more research is needed to determine the causal relationship and pathways between perceived discrimination and health, prompt attention and intervention to address depression among the Chinese diaspora during the COVID-19 pandemic may partially relieve the disease burden caused by the surge of discrimination. Notably, this study provides new perspectives on the different roles of anxiety and depression and the varied roles of social support from different populations in mediation of the discrimination-health relationship specific to the COVID-19 context, indicating the uniqueness of this pandemic with regard to psychology, sociology, and health characteristics.

\section{Declarations}

\section{Ethics approval and consent to participate}

This study was approved by the ethics committee of Wenzhou Medical University [2020-073]. All subjects voluntarily consented to participate in the study.

\section{Consent for publication}

Not applicable. 


\section{Availability of data and materials}

The datasets used and analysed during the current study are available from the corresponding author on reasonable request.

\section{Competing interests}

The authors declare that they have no competing interests.

\section{Funding}

Funding/Support: This project was sponsored by the National Natural Science Foundation of China [71603187].

Role of the Funder/Sponsor: The funder had no role in the design and conduct of the study; collection, management, analysis, and interpretation of the data; preparation, review, or approval of the manuscript; or decision to submit the manuscript for publication.

\section{Author Contributions}

YC is responsible for conceptualization, data curation, formal analysis, investigation, methodology, resources, software, and writing of the original draft.

ZW is responsible for investigation, resources, and writing of the original draft.

WD is responsible for conceptualization, investigation, resources, and reviewing and editing the manuscript.

$\mathrm{JC}$ and $\mathrm{SJ}$ are responsible for investigation and resources.

$\mathrm{XZ}$ is responsible for investigation, and reviewing and editing the manuscript.

$\mathrm{CC}$ is responsible for conceptualization, funding acquisition, resources, supervision, and reviewing and editing the manuscript.

\section{Acknowledgement}

We thank all the participants for their willingness to participate in the study.

\section{References}

1. Aronson E, Aronson J. The social animal. 12 ed. New York: Worth Publishers, Macmillan Learning; 2018.

2. Jones JM. Prejudice and Racism. 2 ed. New York: McGraw-Hill Companies; 1997. 
3. Zhuang G. Distribution and development trend of overseas Chinese. Overseas Chinese Affairs Study. 2010(4).

4. Textor C. Distribution of overseas Chinese 2018, by continent 2020 [Available from: https://www.statista.com/statistics/632779/chinese-population-distribution-overseas-by-continent/.

5. White AIR. Historical linkages: epidemic threat, economic risk, and xenophobia. The Lancet. 2020;395(10232):1250-1.

6. Paradies $\mathrm{Y}$. A systematic review of empirical research on self-reported racism and health. Int $\mathrm{J}$ Epidemiol. 2006;35(4):888-901.

7. Milner A, Franz B, Henry Braddock J. We Need to Talk About Racism-In All of Its Forms-To Understand COVID-19 Disparities. Health Equity. 2020;4(1):397-402.

8. Jylhä M. What is self-rated health and why does it predict mortality? Towards a unified conceptual model. Soc Sci Med. 2009;69(3):307-16.

9. Alvarez-Galvez J, Salvador-Carulla L. Perceived Discrimination and Self-Rated Health in Europe: Evidence from the European Social Survey (2010). PLoS ONE. 2013;8(9):e74252.

10. Borrell LN, Kiefe Cl, Williams DR, Diez-Roux AV, Gordon-Larsen P. Self-reported health, perceived racial discrimination, and skin color in African Americans in the CARDIA study. Soc Sci Med. 2006;63(6):1415-27.

11. Brondolo E, Hausmann LRM, Jhalani J, Pencille M, Atencio-Bacayon J, Kumar A, et al. Dimensions of Perceived Racism and Self-Reported Health: Examination of Racial/Ethnic Differences and Potential Mediators. Ann Behav Med. 2011;42(1):14-28.

12. Pascoe EA, Smart Richman L. Perceived discrimination and health: a meta-analytic review. Psychol Bull. 2009;135(4):531-54.

13. Myers H, Lewis T, Dominguez T. Stress, coping, and minority health: Biopsychosocial perspective on ethnic health disparities. 2003. p. 377-400.

14. Clark R, Anderson NB, Clark VR, Williams DR. Racism as a stressor for African Americans. A biopsychosocial model. Am Psychol. 1999;54(10):805-16.

15. Cuevas AG, Reitzel LR, Cao Y, Nguyen N, Wetter DW, Adams CE, et al. Mediators of discrimination and self-rated health among African Americans. Am J Health Behav. 2013;37(6):745-54.

16. Chen Y, Wang Z, Dong W, Chen Xu JH, Ji Wu S, Zhang X, et al. Perceived discrimination and mental health among the Chinese diaspora during COVID-19. Asian J Psychiatr. 2021;57:102584.

17. Berkman LF. Assessing the physical health effects of social networks and social support. Annu Rev Public Health. 1984;5:413-32.

18. Robb CE, de Jager CA, Ahmadi-Abhari S, Giannakopoulou P, Udeh-Momoh C, McKeand J, et al. Associations of Social Isolation with Anxiety and Depression During the Early COVID-19 Pandemic: A Survey of Older Adults in London, UK. Front Psychiatry. 2020;11:591120.

19. Jang Y, Choi EY, Park NS, Chiriboga DA, Duan L, Kim MT. Cognitive health risks posed by social isolation and loneliness in older Korean Americans. BMC Geriatr. 2021;21(1):123. 
20. Chen D, Yang T-C. The pathways from perceived discrimination to self-rated health: an investigation of the roles of distrust, social capital, and health behaviors. Soc Sci Med. 2014;104:64-73.

21. Darviri C, Fouka G, Gnardellis C, Artemiadis AK, Tigani X, Alexopoulos EC. Determinants of Self-Rated Health in a Representative Sample of a Rural Population: A Cross-Sectional Study in Greece. International Journal of Environmental Research Public Health. 2012;9(3):943-54.

22. Jivraj S. Are self-reported health inequalities widening by income? An analysis of British pseudo birth cohorts born, 1920-1970. J Epidemiol Community Health. 2020;74(3):255-9.

23. Joutsenniemi KE, Martelin TP, Koskinen SV, Martikainen PT, Härkänen TT, Luoto RM, et al. Official marital status, cohabiting, and self-rated health-time trends in Finland, 1978-2001. Eur J Pub Health. 2006;16(5):476-83.

24. Dzúrová D, Drbohlav D. Gender Inequalities in the Health of Immigrants and Workplace Discrimination in Czechia. Biomed Res Int. 2014;2014:1-9.

25. Hong J, Lee J. Decomposing Income-Related Inequalities in Self-Reported Depression and Self-Rated Health Among Married Immigrants in South Korea. International Journal of Environmental Research Public Health. 2019;16(10):1869.

26. Shadmi E, Chen Y, Dourado I, Faran-Perach I, Furler J, Hangoma P, et al. Health equity and COVID-19: global perspectives. International Journal for Equity in Health. 2020;19(1):104.

27. Otu A, Ahinkorah BO, Ameyaw EK, Seidu A-A, Yaya S. One country, two crises: what Covid-19 reveals about health inequalities among BAME communities in the United Kingdom and the sustainability of its health system? International Journal for Equity in Health. 2020;19(1):189.

28. Rabin R, de Charro F. EQ-5D: a measure of health status from the EuroQol Group. Ann Med. 2001;33(5):337-43.

29. Feng YS, Kohlmann T, Janssen MF, Buchholz I. Psychometric properties of the EQ-5D-5L: a systematic review of the literature. Qual Life Res. 2021;30(3):647-73.

30. Bursac Z, Gauss CH, Williams DK, Hosmer DW. Purposeful selection of variables in logistic regression. Source Code Biol Med. 2008;3:17-.

31. Chan KT-K, Tran TV, Nguyen T-N. Cross-Cultural Equivalence of a Measure of Perceived Discrimination Between Chinese-Americans and Vietnamese-Americans. Journal of Ethnic Cultural Diversity in Social Work. 2012;21(1):20-36.

32. Harnois CE, Bastos JL, Campbell ME, Keith VM. Measuring perceived mistreatment across diverse social groups: An evaluation of the Everyday Discrimination Scale. Soc Sci Med. 2019;232:298-306.

33. Kroenke K, Spitzer R, Williams J. The Patient Health Questionnaire-2: Validity of a Two-Item Depression Screener. Medical care. 2003;41:1284-92.

34. Lowe B, Wahl I, Rose M, Spitzer C, Glaesmer H, Wingenfeld K, et al. A 4-item measure of depression and anxiety: validation and standardization of the Patient Health Questionnaire-4 (PHQ-4) in the general population. J Affect Disord. 2010;122(1-2):86-95. 
35. Kocalevent RD, Finck C, Jimenez-Leal W, Sautier L, Hinz A. Standardization of the Colombian version of the PHQ-4 in the general population. BMC Psychiatry. 2014;14:205.

36. Zhang WR, Wang K, Yin L, Zhao WF, Xue Q, Peng M, et al. Mental Health and Psychosocial Problems of Medical Health Workers during the COVID-19 Epidemic in China. Psychother Psychosom. 2020;89(4):242-50.

37. Lubben J, Blozik E, Gillmann G, lliffe S, von Renteln Kruse W, Beck JC, et al. Performance of an abbreviated version of the Lubben Social Network Scale among three European community-dwelling older adult populations. Gerontologist. 2006;46(4):503-13.

38. National Academies of Sciences E. Medicine, Division of B, Social S, Education, Health, et al. Social Isolation and Loneliness in Older Adults: Opportunities for the Health Care System. Washington (DC): National Academies Press (US).

39. Copyright 2020 by the National Academy of Sciences. All rights reserved.; 2020.

40. Hale T, Angrist N, Kira B, Petherick A, Phillips T, Webster S. Variation in Government Responses to COVID-19. 2020.

41. Kim H-Y. Statistical notes for clinical researchers: assessing normal distribution (2) using skewness and kurtosis. Restor Dent Endod. 2013;38(1):52-4.

42. Muthén LK, Muthén BO. Mplus User's Guide. Seventh Edition ed. Los Angeles, CA: Muthén \& Muthén 1998-2012.

43. Hu L-t, Bentler PM. Fit indices in covariance structure modeling: Sensitivity to underparameterized model misspecification. Psychol Methods. 1998;3(4):424-53.

44. Awang Z, editor SEM Made Simple: A Gentle Approach to Learning Structural Equation Modeling2015.

45. Anderson JC, Gerbing DW. Structural Equation Modeling in Practice. A Review Recommended TwoStep Approach. 1988;103(3):411-23.

46. Asparouhov T, Muthén B. Weighted Least Squares Estimation With Missing Data. Mplus Technical Appendix; 2010.

47. Pew Research Center analysis of 2013-2015 American Community Survey (IPUMS). Educational attainment of Chinese population in the U.S., 20152017 [Available from: https://www.pewsocialtrends.org/chart/educational-attainment-of-chinese-population-in-the-u-s/.

48. Todorova ILG, Falcón LM, Lincoln AK, Price LL. Perceived discrimination, psychological distress and health. Sociol Health Illn. 2010;32(6):843-61.

49. Figueroa CA, DeJong H, Mocking RJT, Fox E, Rive MM, Schene AH, et al. Attentional control, rumination and recurrence of depression. J Affect Disord. 2019;256:364-72.

50. Brosschot JF, Pieper S, Thayer JF. Expanding stress theory: prolonged activation and perseverative cognition. Psychoneuroendocrinology. 2005;30(10):1043-9.

51. Tee ML, Tee CA, Anlacan JP, Aligam KJG, Reyes PWC, Kuruchittham V, et al. Psychological impact of COVID-19 pandemic in the Philippines. J Affect Disord. 2020;277:379-91. 
52. Carter SE, Walker RL, Cutrona CE, Simons RL, Beach SRH. Anxiety Mediates Perceived Discrimination and Health in African-American Women. Am J Health Behav. 2016;40(6):697-704.

53. Assari S. Psychiatric Disorders Differently Correlate with Physical Self-Rated Health across Ethnic Groups. J Pers Med. 2017;7(4).

54. Holt-Lunstad J, Smith TW, Uchino BN. Can hostility interfere with the health benefits of giving and receiving social support? The impact of cynical hostility on cardiovascular reactivity during social support interactions among friends. Ann Behav Med. 2008;35(3):319-30.

55. Smith KP, Christakis NA. Social Networks and Health. Ann Rev Sociol. 2008;34(1):405-29.

56. Berkman LF, Glass T, Brissette I, Seeman TE. From social integration to health: Durkheim in the new millennium. Soc Sci Med. 2000;51(6):843-57.

57. Christopher Westland J. Lower bounds on sample size in structural equation modeling. Electron Commer Res Appl. 2010;9(6):476-87.

58. Kalton G. Probability sampling methods for hard-to-sample populations. In: Edwards B, Wolter KM, Bates N, Tourangeau R, Johnson TP, editors. Hard-to-Survey Populations. Cambridge: Cambridge University Press; 2014. pp. 401-23.

\section{Figures}

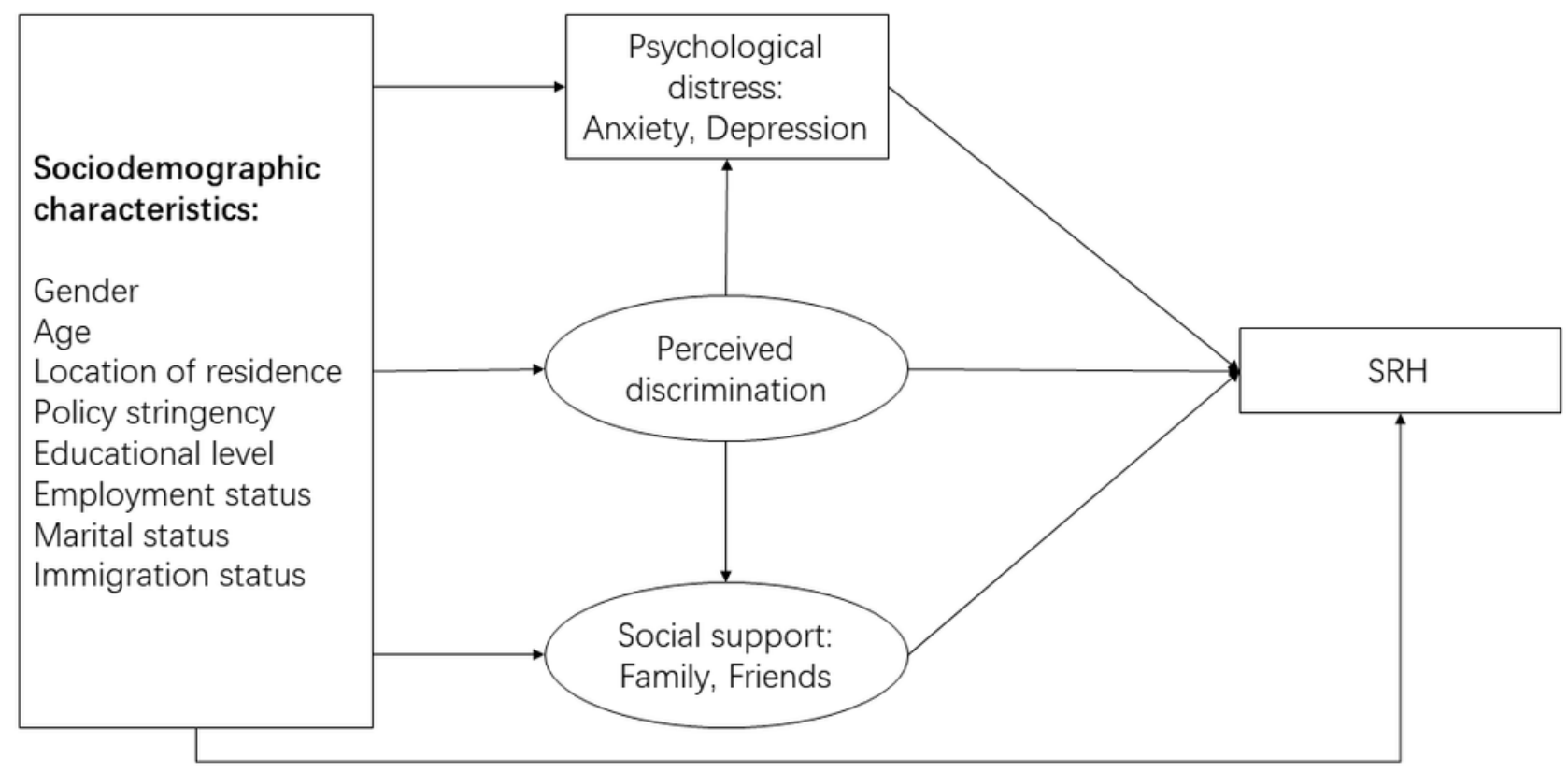

\section{Figure 1}


Conceptual framework and hypotheses Notes: Rectangles indicate the manifest variables, and ovals indicate the latent variables.

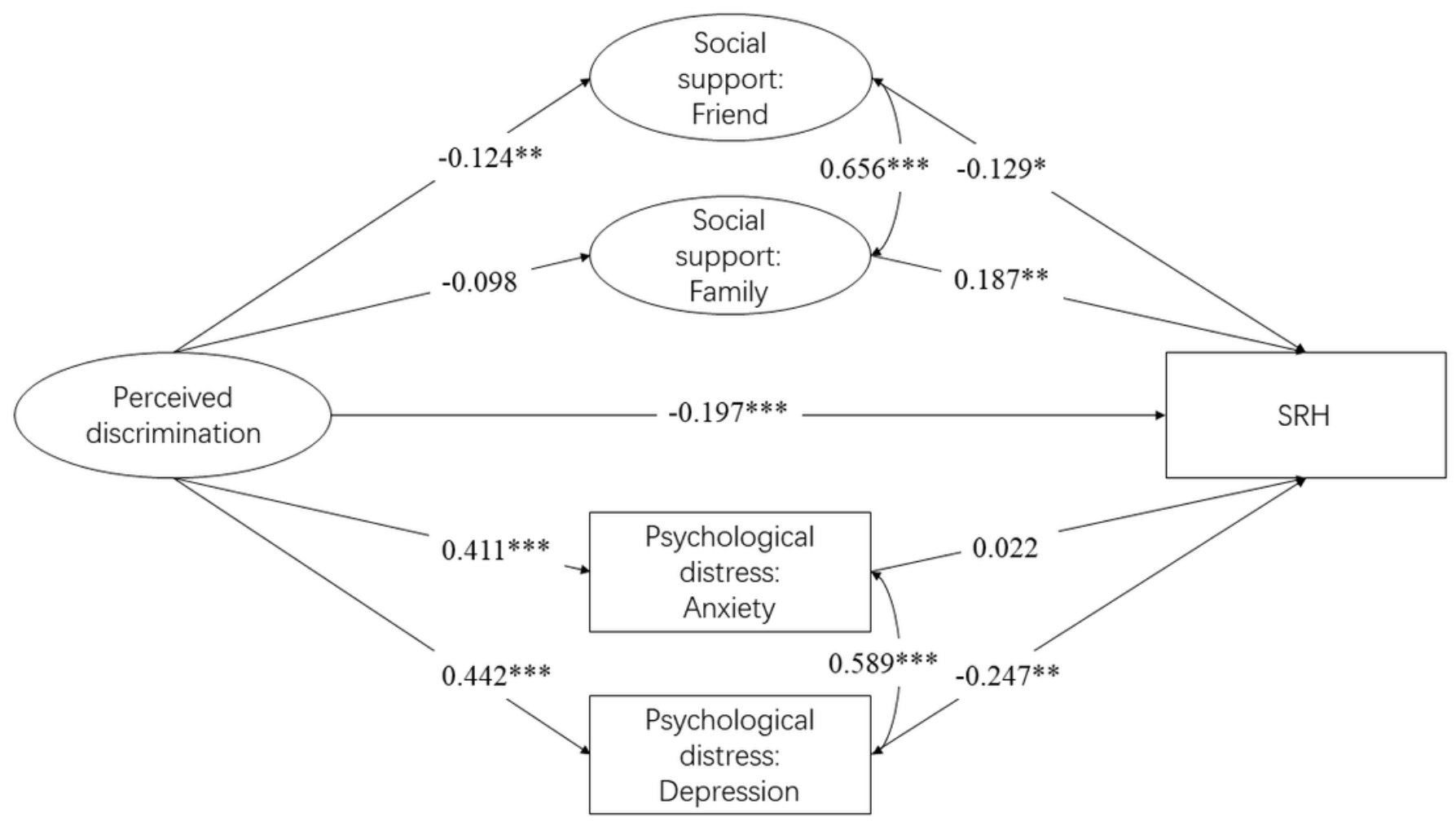

Figure 2

Observed SEM of perceived discrimination on SRH Notes: numbers in the figure along any single-headed arrow are the standardized path coefficients. *, $p<0.05 ; * \star, p<0.01 ; * \star *, p<0.001$

\section{Supplementary Files}

This is a list of supplementary files associated with this preprint. Click to download.

- SupplementaryTable1questionnaire.docx

- SupplementaryTable2normality.docx

- SupplementaryTable3intercorrelation.docx

- SupplementaryTable4internalconsistency.docx 